International Journal of Cancer Studies \& Research (IJCR)

$1 S S N: 2167-9118$

\title{
Serum Lipid Fractions and Renal Cell Carcinoma: A Review of Current Literature
}

Honda $\mathrm{T}$

Review Article

Assistant Professor, Northeastern University, United States.

\section{*Corresponding Author:}

Trent Honda,

Assistant Professor, Northeastern University, United States.

Tel: 562-631-0631

E-mail: t.honda@neu.edu

Received: January 152015

Accepted: February 02, 2015

Published: February 10, 2015

Citation: Honda T (2015) Serum Lipid Fractions and Renal Cell Carcinoma: A Review of Current Literature. Int J Cancer Stud Res. 4(1), 58-62. doi: http:/ /dx.doi.org/10.19070/2167-9118-150008

Copyright: Honda $\mathbf{T}^{\mathcal{O}}$ 2015. This is an open-access article distributed under the terms of the Creative Commons Attribution License, which permits unrestricted use, distribution and reproduction in any medium, provided the original author and source are credited.

\section{Introduction}

Serum Lipid Fractions and Renal Cell Carcinoma Kidney cancer is among the most common human malignancies. In 2008, 273, 518 new cases of kidney cancer were diagnosed throughout the world, representing $2.8 \%$ of the worldwide cancer incidence [1]. In that same year, 116,368 individuals died from kidney cancer [1]. Over $90 \%$ of all kidney cancers are of adenomatous histology (clear cell renal cell carcinoma) and arise from the renal parenchyma, while the remaining $10 \%$ are a heterogeneous group of cancers mainly arising from the renal pelvis tissue [2]. As this smaller group is distinct from the adenomatous tumors both histologically and in terms of known risk factors, this current review will only address clear cell renal cell carcinoma (RCC).

\section{Epidemiology and Risk Factors}

Over the past three decades, the incidence of RCC has increased steadily to the current US incidence rates of 10/100,000 in white men, 4.8/100,000 in white women, 11.5/100,000 in African American men, and 5.7/100,000 in African American women [2]. Worldwide, the incidence varies appreciably with the lowest rates seen in Asia and Latin America (1-2/100,000 men), and the highest in North America and Europe (4.7-15.3/100,000 men) [2].

While the exact cause of the increased incidence of RCCover the last few decades is unknown, evidence suggests that changes in diet and lifestyle have been major contributors. Non-modifiable risk factors include age ( $>50$ years) and male sex [2]. Established modifiable risk factors include: cigarette smoking, obesity, and hypertension [2-7]. Smoking has been found to increase the risk of RCC by $50 \%$ in men, and $20 \%$ in women $[2,8]$. It is hypoth- esized that the major carcinogenic effects of smoking are due to the induction of local tissue hypoxia in the renal parenchyma due to the increased blood carbon monoxide levels which precipitates a carcinogenic molecular cascade in susceptible individuals [2-9]. Obesity is estimated to account for between $30 \%$ and $40 \%$ of RCC cases in the developed world. Numerous potential mechanisms mediating obesity and RCC carcinogenesis have been proposed, including chronic tissue hypoxia, chronic inflammation and lipid peroxidation [10]. These same mechanisms have been hypothesized to mediate the additional risk of RCC attributable to hypertension [9]. Diabetes mellitus has been proposed as a potential risk factor for RCC, however the additional risk of diabetes mellitus independent of the often comorbid obesity and hypertension has not yet been fully elucidated [8]. Protective effects have been observed in some studies between RCC risk and alcohol consumption and physical activity, both with dose-response relationships $[1,2,8]$.

\section{Biological Mechanisms}

Interestingly, most of the established and suspected risk factors for RCC are also established risk factors for abnormal plasma lipid levels, raising the possibility that plasma lipid levels might be an effect modifier for RCC risk [11]. This association would not be unique to RCC; prostate, breast and colorectal cancers have all been found to have significant risks associated with perturbations in serum lipid levels [12-14]. As early as the 1960s, it was observed that the glassy, clear cellular appearance distinct to RCC was caused by an excess of cystoplasmic lipid,mainly in the form of esterified cholesterol [15]. While early studies suggested that this excess cholesterol storage was due to increased intracellular lipid production, more recent analyses have determined that a loss of activity in the VHL tumor suppressor gene causes an increase in the expression of the very low-density lipoprotein receptor (VLDL-R) on RCC cells, resulting in an increase in cholesterol ester uptake from the systemic circulation, with subsequent growth of adipose deposits inside the renal tubular epithelium $[15,16]$. Genetic variations in apolipoprotein E structure, proteins, which bind to the VLDL-R in the process of cellular cholesterol ester uptake, also likely impact the rate of lipid uptake by renal cells and at least one study has shown differential risks of RCC based upon apolipoprotein $\mathrm{E}$ locus variations $[17,18]$.

It is hypothesized that hyperplasia and hypertrophy induced by increased cholesterol uptake in kidney cells increases the metabolic rate in the individual capillary beds feeding this tissue, which subsequently leads to a relative tissue hypoxia. In response to this oxygen deficit, the tissues up-regulate production of hypoxia-inducible factor-1- $\alpha$ (HIF $-1 \alpha)$ [6]. HIF-1 $\alpha$ suppresses cellular apop- 
tosis and up-regulates expression of vascular endothelial growth factor (VEGF) [6]. Suppression of cellular apoptotic mechanism permits abnormal cells to proliferate, and VEGF promotes local blood vessel proliferation (neoangiogenesis), both of which are characteristic of RCC tumor growth.

The inverse associations reported in some epidemiologic studies between RCC and increased physical activity and alcohol consumption could also potentially be explained by the impact of these behaviors on serum lipid fractions. Physical activity has been shown to decrease plasma triglycerides, VLDL and low-density lipoproteins (LDL) [11]. Reductions in these lipid fractions could potentially decrease the substrate available for cholesterol ester uptake by RCC cells from the systemic circulation, thereby decreasing the rate of abnormal lipid accumulation in RCC cells. Likewise, alcohol ingestion in moderate amounts is associated with increases in high-density lipoprotein (HDL), the lipid fraction associated with reverse cholesterol transport from peripheral tissues (such as the renal parenchymal vasculature) back to the liver [19-21]. One study found a modestly significant inverse relationship between high serum HDL $(>55.3 \mathrm{mg} / \mathrm{dl})$ as compared to low serum HDL $(<36.2 \mathrm{mg} / \mathrm{dl})$ and total cancer incidence $(\mathrm{RR}$ $0.89,95 \%$ CI $0.83-0.97, \mathrm{P}=.01)$ which lends some modest, indirect support to the hypothesis that alcohol-induced alterations in lipid fractions might explain the inverse association between RCC and increased alcohol consumption [22].

Despite the evidence that abnormal lipid metabolism is a potential factor in RCC carcinogenesis and tumor progression, little research on the direct association between RCC and lipid levels has been undertaken. It is the intent of this literature review to summarize the available studies evaluating the association between RCC and serum lipid levels and to identify areas in need of further research.

\section{Methods}

Pubmed, Medline and CINHAL databases were searched for epidemiological studies with the following search terms: Dyslipidemia AND renal cell carcinoma, lipoprotein AND renal cell carcinoma, statin AND renal cell carcinoma, and lipid AND renal cell carcinoma. Nineteen relevant articles were identified. Two articles explored potential biological mechanisms for the association between lipid disorders and RCC and are included in the introductory section above. Three papers published only as abstracts and two editorial responses were excluded. The remaining twelve articles are summarized below.

\section{Literature Review}

The available literature on RCC risk and lipid disorders mainly addresses two related questions:

1. what is the association of RCC with the various lipid fractions and

2. does pharmacologic treatment with anti-cholesterolemic medication ameliorate RCC risk?

Herein, the literature relevant to each of these questions is addressed in turn.

\section{What is the association of RCC with the various lipid fractions?}

The association of RCC with lipid fraction levels has been investigated in six studies. Zhang G. et al.reported statistically significant associations between high LDL levels and RCC in a case-control study including 550 histologically confirmed RCC cases and 570 age and sex-matched hospital controls in a Chinese population [23]. To control for influence from know RCC risk factors, odds ratios adjusted for age, gender, BMI, smoking status, hypertension and diabetes were calculated. Insignificant associations were observed between borderline high total cholesterol $\left(\mathrm{OR}_{\text {adj }} 0.964\right.$, 95\% CI 0.711-1.305, $\mathrm{P}=0.534)$ and high total cholesterol ( $O \mathrm{R}_{\text {adj }}$ 1.258, 95\% CI 0.694-2.280, $\mathrm{P}=0.534)$. Odds for borderline high triglyceride levels (OR $1.091,95 \%$ CI $0.735-1.620)$ and high triglyceride levels $\left(\mathrm{OR}_{\mathrm{adj}} 1.276,95 \% \mathrm{CI} 0.861-1.891\right)$ were insignificantly associated with RCC risk [23]. Odds ratios for LDL and RCC were both statistically significant and much stronger than for either total cholesterol or triglycerides $\left(\mathrm{OR}_{\text {adj }}\right.$ borderline high LDL 1.994, 95\% CI 1.290-3.084, P=0.002; OR adj high LDL 9.311, 95\% CI 3.426-25.311, $\mathrm{P}=0.002$ ) [23]. Odds ratios for HDL were insignificantly associated with RCC at any level of exposure stratification [23]. Interestingly, odds ratios between LDL and RCC increased appreciably as the tumor stage increased. (T1 ORadj5.636, 95\% CI 1.995-15.924, P=0.001; T2 OR $\mathrm{adj}_{31.043,95 \%}$ CI 7.489-128.678, P=0.228; T3-T4 OR adj 28.915 , 95\% CI 6.221134.394, $\mathrm{P}=0.479)$ [23]. While the strength of association was most dramatic in the high LDL stratification at high tumor grades, the findings were not statistically significant as the sample size relevant to these strata was small. Additional weaknesses of the study include the lack of a clear temporal relationship between elevated LDL levels and RCC development, and a lack of assessment or adjustment for anti-cholesterolemic medication status.

A second recent case-control study in a Chinese population reported findings contradictory to those above [24]. Cases $(n=248)$ were selected from a hospitalized population with histologically confirmed RCC, and were matched on a 1:2 ratio with non-hospitalized community controls $(n=496)$ matched for age and gender [24]. The authors report decreased risks of RCC associated with: elevated total cholesterol (HR 0.642, 95\% CI 0.535-0.769), elevated LDL (HR 0.745, 95\% CI 0.613-0.905), and elevated HDL (HR 0.175 , 95\% CI 0.095-0.320). Elevations in triglycerides did not meet statistical significance (HR 1.305, 95\% CI 0.922-1.846) [24]. This study differs in a number of ways from that conducted by Zhang G. et al and these differences may have influences the differential findings. First, the controls for this study were selected from the general population, while those in the prior study were hospital-based controls. Second, the authors did not control for alcohol consumption, which is known to have an inverse relationship with RCC risk. Either or both of these differences could have contributed to a substantial introduction of bias in this study.

In a large, cohort study in an Austrian population, Ulmer et al. investigated the association between elevated triglycerides and numerous cancers [25]. The 156,153 subjects were followed for an average of 10.6 years. Triglyceride levels were assessed annually for those continually enrolled in the study, and the data were linked to the state cancer registry. During the follow-up period, 216 kidney cancers were identified in study participants. Hazard 
ratios ranged from 0.81 in the lowest triglyceride quartile $(95 \%$ CI $0.51-1.280, \mathrm{p}=0.105)$ to 1.27 in the highest triglyceride quartile (95\% CI 0.81-1.97, $\mathrm{P}=0.105$ ) [25]. In no quartile was the association statistically significant. This study is limited in a number of ways. Primarily, the exclusion of analysis for any other lipid fraction significantly limits the scope of the findings in terms of the current literature review. Additionally, the authors did not stratify the incident kidney cancers by histologic type, so the associations with RCC specifically are unable to be assessed. Additional problems with risk factor assessment and adjustment also might have biased the results. First, the authors did not control for alcohol consumption, which is both a known cause of hypertriglyceridemia and inversely associated with RCC risk in some studies; second, the authors self-identified that a differential misclassification of smoking status might have occurred due to design flaws [25]. Lastly, anti-cholesterolemic medication status was not ascertained.

Triglyceride levels were significantly associated with RCC in a large Swedish cohort study [5]. Hemelrijck et al. examined associations between kidney cancer, triglycerides and total cholesterol in the full $(n=542,924)$ cohort, and association between kidney cancer and a number of other lipid fractions [HDL, LDL, apolipoprotein-a (Apo-a) and apolipoprotein-b (Apo-b)] in a subgroup for whom this data was available $(n=85,621)$. Over a mean follow-up period of 13 years, 958 kidney cancers were observed. Triglycerides were reported to have an association with RCC risk in the $2^{\text {nd }}$ (HR 1.25, 95\% CI 0.99-1.60), $3^{\text {rd }}$ (HR 1.29, 95\% CI1.01-1.66), and $4^{\text {th }}$ (HR 1.66, 95\% CI1.30-2.13) quartiles of triglyceride exposure as compared to the $1^{\text {st }}$ quartile $\left(\mathrm{P}_{\text {trend }}<0.001\right)[5]$. No significant associations with any other lipid fraction were observed. This study is limited by a number of factors. First, while the cohort is large, certain characteristics of the cohort suggest that it is not representative of the larger population. The prevalence of obesity reported in the study population was $1.25 \%$, which is significantly lower than the average values in the US and Europe. Additionally, during the study period all cause mortality in the cohort was $14 \%$ lower than in the general Swedish population. These findings suggest that the cohort in the study was healthier than the general population, and call in to question the external validity of the study. The study is limited further as it did not assess for a number of variables that are known to affect RCC risk, including: smoking, hypertension status, anti-cholesterolemic medication status, and tumor grade. Lastly, no differentiation between the various kidney cancer histologies was reported.

Haggstrom et al. also investigated the association of lipid fractions with RCC in a large cohort of Norwegian, Austrian and Swedish men and women [4]. 560,388 men and women were followed for an average of 10 years, during which 592 men and 263 women developed RCC. The authors reported statistically significant correlations between triglyceride levels in the highest quintile of exposure for men (HR 2.38, 95\% CI 1.37-4.15, $\mathrm{p}=.0002$ ) but not for women [4]. The authors only investigated one other lipid fraction (total cholesterol) and found no association with RCC. Age, smoking status, BMI, and hypertension status were controlled for in the analysis, but stratification based upon alcohol exposure was not completed which could have biased the results. Additional limitations of this study include the lack of assessment for anti-cholesterolemic medication status.

Ahn et al. analyzed data from 29, 093 men enrolled in the Alphatocopherol, Beta Carotene Cancer Prevention study in Finland for associations between numerous cancers and HDL and total cholesterol [22]. During a median of 14.9 years of follow-up, 290 cases of kidney cancer (undifferentiated by histopathology) occurred. No statistically significant association was found between kidney cancer development and total cholesterol $(\mathrm{P}=0.08)$ or HDL $(\mathrm{P}=0.20)$ in any quartile of exposure. However, an inverse relationship was reported between high serum HDL $(>55.3 \mathrm{mg} /$ $\mathrm{dl}$ ) and total cancer incidence (RR 0.89, 95\% CI 0.83-0.97, P=.01). This study is limited as no assessment of anti-cholesterolemic medication status was performed; the generalizability of the study is limited as all study subjects were smokers and men.

\section{Does pharmacologic treatment with anti-cholester- olemic medication ameliorate RCC risk?}

Lipid disorders are often treated to reduce the risk of coronary artery disease and other vascular diseases. The mainstay of dyslipidemia treatments is a group of compounds that reduce cholesterol synthesis by inhibition of 3-hydroxy-3-methylglutarylcoenzyme Areductase (statins). Some recent studies have found that statin medication use is inversely associated with RCC risk and tumor progression.

In a large, nested case-control study of United States Veterans Affairs data, Khurana et al. found a statistically significant risk reduction for RCC among those taking statins [26]. 483, 733 individuals from eight states were included in the study. Statin use was reported in 164, 441 (34\%) and RCC was present in 1446 $(0.3 \%)$. After adjusting for age, gender, BMI and smoking status, the statin group was found to have a $48 \%$ risk reduction for RCC $\left(\mathrm{OR}_{\text {adj }} 0.52,95 \%\right.$ CI $\left.0.45-0.60\right)$ [26]. This study is limited in its external validity as the cohort is composed entirely of veterans and represents only southern US states (Florida, Alabama, Mississippi, Louisiana, Arkansas, Missouri, Oklahoma and Texas). Additionally, the study did not collect data on lipid fractions, which precludes analysis of the direct effects of lipid levels on RCC risk. Lastly, statin duration, dose and type and alcohol exposure were not controlled for which could have biased the study results.

Liu et al. found a small but significant risk reduction associated with statin use by pooling data from the Nurses Health Study and the Health Professions Follow-up Study [27]. The authors followed 80,782 women for an average of 14 years, and 37,869 men for an average of 16 years. Twenty-two percent of women, and $23 \%$ of men reported statin use. During the follow-up time period, 277 cases of RCC were reported (164 women, 113 men). After controlling for smoking, BMI, hypertension, diabetes, NSAID use, intake of fruits/vegetables and alcohol, a borderline statistically significant risk reduction was observed in women (RR $0.68,95 \%$ CI $0.46-1.00)$ and an insignificant increased risk was observed in men (RR 1.17, 95\% CI 0.75-1.82) [27]. The strongest risk reductions were seen in the subset of women without a history of hypertension (RR $0.28,95 \%$ CI 0.09-0.92).

While reductions in plasma cholesterol levels are one potential way in which statins could exert antineoplastic effects in RCC, other molecular effects of these medications have also been described which could explain the observed antineoplastic effects of statins. Two studies report that statins inhibit RCC cell proliferation, cellular migration and promote cellular apoptosis by suppressing the $\mathrm{AKT} /$ mammalian target of rampamycin (AKT/ mTOR) pathway and Interleukin-6 in RCC tumor cells which acts 
to suppress neoangiogenesis [28,29]. Irrespective of the actual method of tumor cell suppression (via indirect lipid suppression or direct molecular pathways in the RCC cells) the proven safety and efficacy of statins would make them an important investigational treatment for renal cell carcinoma.

Two studies investigated the effect of statin therapy on RCC tumor recurrence and progression after surgical resection [30,31]. Choi et al. evaluated 115 men who had undergone partial or radical nephrectomy for non-metastatic RCC for recurrence or progression of the cancer. Retrospective analysis of health records demonstrated that $21(18 \%)$ patients were on statins, and $94(82 \%)$ were not. While those who began statins after surgery were excluded from the statin group, no information on statin dose, type, duration of treatment or lipid levels were included in the analysis. Differences in recurrence-free survival duration and progression-free survival duration did not reach statistical significance ( $p=0.586$ and $p=0.307$, respectively) and the authors concluded that statins do not have a marked effect on recurrence or progression [31]. This study is significantly limited by small size and a lack of standardization of statin exposure. The authors also did not measure plasma lipid levels, which limits the utility of this information in analyzing the associations of lipid disorders and RCC.

A similar study by Hamilton et al. found reduced recurrence and progression of RCC associated with statin use. 2,608 patients with localized RCC treated surgically were identified as the study population [30]. Medical record review determined which subjects were taking statins prior to their surgical resection $(n=708)$ and which were not $(n=1900)$. Unlike the study by Choi et al., detailed information on statin therapy type and duration was collected which permitted stratification by statin exposure. Statin use at surgery was associated with a statistically significant 33\% decreased risk of progression (HR 0.67, 95\% CI 0.47-0.94, $\mathrm{p}=0.028)$. No significant associations were found between statin dose or type and progression, although the vast majority of patients were taking simvastatin $(n=33 \%)$ or atorvastatin $(46 \%)$ leaving very small numbers of patients on each of the remaining 5 statin types which could have impacted the ability for the differential effects of those statins to reach statistical significance. Alternatively, and importantly, as the statin therapy was likely initiated to decrease the risk of cardiovascular disease via correction of dyslipidemia, the risk reduction reported might be due to the effect of the statin on the lipid levels and not on the exposure to a statin per se. Assuming each individual had his/her statin level titrated to a lipid stratum that confers similar risk to all individuals, this might explain why no significant difference between statin type/dose was observed. Unfortunately, this study, like that by Choi et al., focused on examining the direct effects of statins as tumor suppressors and not on an indirect effects via alterations in lipid levels and therefore no information on serum lipid levels was collected or included in the analysis [30].

\section{Discussion}

Relatively few studies investigate the relationship between RCC and lipid levels, and their results present a fragmented and often contradictory picture. Most are limited due to methodological flaws that significantly impact their internal and/or external validity. Two case-control studies in Chinese populations came to contradictory conclusions with Zhang G. et al. reporting a sig- nificant increased risk of RCC associated with elevated LDL, and Zhang C. et al. reporting a significant decreased risk associated with elevated LDL $[23,24]$. Of the four European cohort studies, one investigated only the association of RCC with triglycerides [25], one with total cholesterol and HDL [22], one with triglycerides and total cholesterol[4], and one with multiple lipid fractions (LDL, HDL, Total cholesterol, triglycerides, Apo-a, Apo-b) [5]. All of the studies found increase risks associated with elevated triglyceride levels, although only three of the four reached statistical significance. No other lipid fraction was found to be significantly associated with RCC risk, however only triglycerides were investigated in every study, and there were only four studies. This failure to overlap primary variables between these studies complicates any attempt to draw together the study results to make meaningful conclusions. Importantly, there are no studies of US populations that investigate measured lipid fractions and RCC risk despite the US having among the highest incidence of RCC and RCC risk factors in the world.

Four studies assess the association between RCC and statin therapy. One large nested case-control study analyzed US Veterans Affairs data and found a profound risk reduction among those taking statins [26]. However, a second study pooling data from two large prospective cohort studies found a statistically significant risk reduction only in women without a history of hypertension [27]. Two studies investigated whether statin therapy decreases the risk of RCC tumor recurrence and progression and came to conflicting results $[30,31]$. As the risk of RCC in the general population is low, and dyslipidemia is a known risk factor for, and generally treated for, the much more prevalent cardiovascular diseases, the effect of pharmacologic manipulation of lipids as a method of decreasing tumor recurrence or progression (as opposed to primary prevention of RCC) is perhaps the most important clinical question that remains to be answered. Unfortunately, none of the studies investigating the associations of statins and RCC collected, reported or analyzed lipid fraction levels which precludes the ability to rule-in or rule-out the possibility that statins impact RCC risk by decreasing lipid levels. Instead these studies theoretically oriented themselves around the hypothesis that statins have antineoplastic properties independent of their effect on lipids.

The importance of further delineating the effect of statin therapy on tumor progression and recurrence cannot be overstated. Currently, a number of medications are approved for the treatment of metastatic RCC, all of which cost between $\$ 111,000$ and $\$ 196,000$ per year of treatment, and are associated with a range of serious adverse drug reactions [32]. By contrast, statins are a relatively safe, inexpensive medication and, if their effects on RCC progression are indeed due to indirect manipulation of serum lipids, they could be easily titrated for clinical efficacy. Until further research on the matter is completed, however, these possibilities remain entirely speculative.

\section{Conclusion}

There is compelling evidence that serum lipid fraction levels are a potential factor in RCC carcinogenesis and tumor progression. Unfortunately, the dearth of available information is further complicated by the fragmentary nature and the methodological limitations of the available studies. Further delineation of the relationship between lipid fractions and RCC is important, as lipids would be a safe and inexpensive risk factor to pharmacologically modify. 


\section{References}

[1]. Boyle P, B. Levin (2008) World cancer report 2008. IARC Press, International Agency for Research on Cancer, France.

[2]. Wong-Ho Chow, L.M.D, Susan S.Devesa (2010) Epidemiology and risk factors for kidney cancer. Nat Rev Urol 7(5): 245-257.

[3]. Bergstrom A (2001) Obesity and renal cell cancer--a quantitative review. Br J Cancer 85(7): 984-90.

[4]. Haggstrom C (2013) Metabolic factors associated with risk of renal cell carcinoma. PLoS One 8(2): e57475.

[5]. Van Hemelrijck M (2012) The interplay between lipid profiles, glucose, BMI and risk of kidney cancer in the Swedish AMORIS study. Int J Cancer 130(9): 2118-28.

[6]. Zhang G.M, Y. Zhu, D.W. Ye (2014) Metabolic syndrome and renal cell carcinoma. World J Surg Oncol 12: 236

[7]. Bhaskaran K (2014) Body-mass index and risk of 22 specific cancers: a population-based cohort study of 5.24 million UK adults. Lancet 384(9945): 755-65.

[8]. Setiawan V.W (2007) Risk factors for renal cell cancer: the multiethnic cohort. Am J Epidemiol 166(8): 932-40.

[9]. Sharifi N, W.L Farrar (2006) Perturbations in hypoxia detection: a shared link between hereditary and sporadic tumor formation?. Med Hypotheses 66(4): 732-5.

[10]. Klinghoffer Z (2009) Obesity and renal cell carcinoma: epidemiology, underlying mechanisms and management considerations. Expert Rev Anticancer Ther 9(7): 975-87.

[11]. National Cholesterol Education Program Expert Panel on Detection E. and A. Treatment of High Blood Cholesterol in, Third Report of the National Cholesterol Education Program (NCEP) Expert Panel on Detection, Evaluation, and Treatment of High Blood Cholesterol in Adults (Adult Treatment Panel III) final report. Circulation (2002). 106(25): 3143-421.

[12]. Kucharska-Newton A.M (2008) HDL-cholesterol and incidence of breast cancer in the ARIC cohort study. Ann Epidemiol 18(9): 671-7.

[13]. Mittal A (2011) Role of hypercholesterolemia in prostate cancer--case control study from Manipal Teaching Hospital Pokhara. Asian Pac J Cancer Prev 12(8): 1905-7.

[14]. Wulaningsih W (2012) Serum Lipids and the Risk of Gastrointestinal Malignancies in the Swedish AMORIS Study. J Cancer Epidemiol 2012: 792034.

[15]. Gebhard R.L (1987) Abnormal cholesterol metabolism in renal clear cell carcinoma. J Lipid Res 28(10): 1177-84.

[16]. Sundelin J.P (2012) Increased expression of the very low-density lipoprotein receptor mediates lipid accumulation in clear-cell renal cell carcinoma. PLoS One 7(11): e48694.

[17]. Knouff C (1999) Apo E structure determines VLDL clearance and atherosclerosis risk in mice. J Clin Invest 103(11): 1579-86.

[18]. Moore L.E (2009) Apolipoprotein E/C1 locus variants modify renal cell carcinoma risk. Cancer Res 69(20): 8001-8.

[19]. Chiva-Blanch G (2014) Effects of alcohol and polyphenols from beer on atherosclerotic biomarkers in high cardiovascular risk men: A randomized feeding trial. Nutr Metab Cardiovasc Dis 25(1):36-45.

[20]. Galan I (2014) Alcohol drinking patterns and biomarkers of coronary risk in the Spanish population. Nutr Metab Cardiovasc Dis 24(2): 189-97.

[21]. Hao G (2014) Relationship Between Alcohol Consumption and Serum Lipid Profiles Among Middle-Aged Population in China: A Multiple-Center Cardiovascular Epidemiological Study. Angiology pii: 0003319714549557, [Epub ahead of print].

[22]. Ahn J (2009) Prediagnostic total and high-density lipoprotein cholesterol and risk of cancer. Cancer Epidemiol Biomarkers Prev 18(11): 2814-21.

[23]. Zhang G.M (2014) Prevalence of dyslipidaemia in patients with renal cell carcinoma: a case-control study in China. BJU Int 113(5b): E75-81.

[24]. Zhang C (2013) Association of dyslipidemia with renal cell carcinoma: a 1 ratio2 matched case-control study. PLoS One 8(3): e59796.

[25]. Ulmer H (2009) Serum triglyceride concentrations and cancer risk in a large cohort study in Austria. Br J Cancer 101(7): 1202-6.

[26]. Khurana V, G. Caldito, M. Ankem (2008) Statins might reduce risk of renal cell carcinoma in humans: case-control study of 500,000 veterans. Urology 71(1): 118-22.

[27]. Liu W, T.K. Choueiri, E. Cho (2012) Statin use and the risk of renal cell carcinoma in 2 prospective US cohorts. Cancer 118(3): 797-803.

[28]. Woodard J (2008) Statin-dependent suppression of the Akt/mammalian target of rapamycin signaling cascade and programmed cell death 4 up-regulation in renal cell carcinoma. Clin Cancer Res 14(14): 4640-9.

[29]. Fang Z (2013) Simvastatin inhibits renal cancer cell growth and metastasis via AKT/mTOR, ERK and JAK2/STAT3 pathway. PLoS One 8(5): e62823.

[30]. Hamilton R.J (2014) The association between statin medication and progression after surgery for localized renal cell carcinoma. J Urol 191(4): 914-9.

[31]. Choi S.K (2013) Effects of statins on the prognosis of local and locally advanced renal cell carcinoma following nephrectomy. Mol Clin Oncol 1(2): 365-368.

[32]. Daniel M. Geynisman, J.C Hu, Lei Liu, Ya-Chen Tina Shih (2014) Treatment Patterns and Costs for Metastatic Renal Cell Carcinoma Patients With Private Insurance in the United States. Clinical Genitourinary Cancer pii: S1558-7673(14)00206-7, [Epub ahead of print]. 